\section{Oseltamivir a winner in fight against influenza virus infections}

Oseltamivir is a cost-effective strategy in the fight against influenza virus infections, according to two US studies.

In the first study, researchers used a stochastic simulation model to determine the cost utility, from a societal perspective, of alternative pandemic influenza mitigation strategies in the US. ${ }^{1}$ In all, 17 strategies were compared including no intervention, targeted antiviral prophylaxis (TAP) alone and in combination with school closure using oseltamivir, as well as prevaccination with low efficacy vaccines. TAP included household targeted antiviral postexposure prophylaxis (HTAP) and prophylaxis in the full set of contact groups for an index case (full targeted postexposure prophylaxis [FTAP]). The time horizon was 6 months.* Quality-adjusted lifeyears (QALYs) were calculated and a cost-utility analysis was performed, calculating costs per QALY gained (2006 values). The FTAP strategy dominated all single strategies (i.e. it was most effective and least costly) and most combination strategies which were therefore eliminated from further analysis. Eliminating all dominated interventions left only FTAP, FTAP plus school closure, and prevaccination plus school closure for comparison [see table].

\begin{tabular}{|c|c|c|c|}
\hline \multicolumn{4}{|c|}{$\begin{array}{l}\text { Incremental cost-utility analysis for noneliminated } \\
\text { strategies }\end{array}$} \\
\hline Strategy & FTAP & $\begin{array}{l}\text { FTAP + } \\
\text { school } \\
\text { closure }\end{array}$ & $\begin{array}{l}\text { Prevaccination } \\
\quad+\text { school } \\
\text { closure }\end{array}$ \\
\hline Total cost ${ }^{\mathrm{a}}$ & 0.12 & 2.73 & 2.73 \\
\hline Incremental cost ${ }^{a}$ & - & 2.48 & 2.50 \\
\hline QALYs $^{\mathrm{b}}$ & 21352 & 21403 & 21403 \\
\hline Incremental QALYs ${ }^{\mathrm{b}}$ & - & 51 & 51 \\
\hline $\begin{array}{l}\text { Incremental cost- } \\
\text { utility ratio (\$US) }\end{array}$ & - & 48472 & 48638 \\
\hline \multicolumn{4}{|c|}{$\begin{array}{l}\mathrm{a} \text { in } \$ \text { US million per } 1000 \text { population } \\
\mathrm{b} \text { per } 1000 \text { population }\end{array}$} \\
\hline
\end{tabular}

Compared to FTAP without school closure, FTAP plus school closure or prevaccination plus school closure gains 51 QALYs but increases the total cost by around $\$$ US2.5 billion for a population of 1000 , driven mainly by extensive work loss for teachers and carers. The incremental cost-utility ratio for either strategy compared with FTAP alone is approximately \$US48 500/ QALY gained.

In the second study, ${ }^{* *}$ researchers used a decisionanalysis model (1-year time horizon) to determine the cost effectiveness of treating influenza-like illness with oseltamivir compared with usual care (symptomatic treatment with nonprescription drugs) in four patient populations: high-risk adolescents and adults, elderly, healthy adolescents and adults, and children. ${ }^{2}$ The model generated outcomes for both a societal and payer perspectives, using efficacy data from clinical trials, presented in 2006 values. Direct costs were based on resource use and indirect costs were based on time lost from work due to illness and premature mortality. From the societal perspective, oseltamivir was cost effective in all populations, particularly in the high-risk group. For high-risk adolescents and adults, the direct cost per patient treated was \$US349 for usual care versus
\$US374 for oseltamivir, giving an incremental cost per healthy day gained of \$US15.09 and an incremental cost per QALY gained \$US5388. From a payer perspective, oseltamivir was cost effective in all population groups. For high-risk adolescents and adults, the cost per patient treated was around \$US344 for usual care versus \$US345 for oseltamivir, giving an incremental cost per healthy day gained of \$US1.08 and an incremental cost per QALY gained of \$US387.

* reflecting the time until a fully-matched vaccine would be available in sufficient quantities to effectively protect the population

** funded by Roche Laboratories

1. Sander B, et al. Economic evaluation of influenza pandemic mitigation strategies in the United States using a stochastic microsimulation transmission model. Value in Health 12: 226-233, No. 2, Mar-Apr 2009.

2. Talbird SE, et al. Cost-effectiveness of treating influenzalike illness with oseltamivir in the United States. American Journal of Health-System Pharmacy 66: 469-480, No. 5, 1 Mar 2009. 